\title{
Shigella sonnei
}

National Cancer Institute

\section{Source}

National Cancer Institute. Shigella sonnei. NCI Thesaurus. Code C86747.

A species of facultatively anaerobic, Gram negative, rod shaped bacteria in the phylum

Proteobacteria. This species is indole and citrate negative, ferments mannitol and

ornithine and is beta-D-galactosidase positive. S. sonnei is found in the human

gastrointestinal system and is pathogenic, being a causative agent of shigellosis. 